T. Akita

Nagoya Math. J.

Vol. 165 (2002), 1-22

\title{
NILPOTENCY AND TRIVIALITY OF MOD $p$ MORITA-MUMFORD CLASSES OF MAPPING CLASS GROUPS OF SURFACES
}

\author{
TOSHIYUKI AKITA*
}

\begin{abstract}
This paper is concerned with $\bmod p$ Morita-Mumford classes $e_{n}^{(p)} \in$ $H^{2 n}\left(\Gamma_{g}, \mathbb{F}_{p}\right)$ of the mapping class group $\Gamma_{g}$ of a closed oriented surface of genus $g \geq 2$, especially triviality and nontriviality of them. It is proved that $e_{n}^{(p)}$ is nilpotent if $n \equiv-1(\bmod p-1)$, while the stable $\bmod p$ Morita-Mumford class $e_{n}^{(p)} \in H^{2 n}\left(\Gamma_{\bullet}, \mathbb{F}_{p}\right)$ is proved to be nontrivial and not nilpotent if $n \not \equiv-1$ $(\bmod p-1)$. With these results in mind, we conjecture that $e_{n}^{(p)}$ vanishes whenever $n \equiv-1(\bmod p-1)$, and obtain a few pieces of supporting evidence.
\end{abstract}

\section{$\S 1$. Introduction}

Let $\Sigma_{g}$ be a closed oriented surface of genus $g \geq 2$ and let $\Gamma_{g}$ be the mapping class group of $\Sigma_{g}$. The cohomology of $\Gamma_{g}$ is one of the most important objects in topology as well as in algebraic geometry. Indeed, any cohomology classes of $\Gamma_{g}$ can be considered as characteristic classes of surface bundles, while the rational cohomology of $\Gamma_{g}$ is naturally isomorphic to that of the moduli space of compact Riemann surfaces of genus $g$ (see [11], [34] for instance).

Morita [28] and Mumford [35] independently introduced a series of certain cohomology classes $e_{n} \in H^{2 n}\left(\Gamma_{g}, \mathbb{Z}\right)(n \geq 0)$ which are called MoritaMumford classes of $\Gamma_{g}$ (see $\S 2$ for the definition). Concerning rational Morita-Mumford classes, Miller [27] and Morita [28] independently proved that the natural homomorphism

$$
\mathbb{Q}\left[e_{1}, e_{2}, e_{3}, \ldots\right] \longrightarrow H^{*}\left(\Gamma_{g}, \mathbb{Q}\right)
$$

Received August 23, 2000.

2000 Mathematics Subject Classification: Primary 55R40, 57R20; Secondary 14H37, $20 \mathrm{~J} 06$.

*The author is supported by Grand-in-Aid for Encouragement of Young Scientists (No. 12740030), the Ministry of Education, Science, Sports and Culture. 
is injective in dimensions less than $2 g / 3$ (see [1] for an elementary proof of this fact). It is conjectured that the above map is actually an isomorphism in dimensions less than $2 g / 3$, and there are many pieces of evidence which support the conjecture (see [34]). On the contrary, little is known about integral or mod $p$ Morita-Mumford classes apart from a recent work of Kawazumi and Uemura [21] which shows that integral Morita-Mumford classes can play an interesting rôle in a study of cohomological properties of finite subgroups of $\Gamma_{g}$. For further results concerning Morita-Mumford classes, see [9], [11], [30], [31], [32], [33], [34] and references therein.

The purpose of this paper is to investigate $\bmod p$ Morita-Mumford classes $e_{n}^{(p)} \in H^{2 n}\left(\Gamma_{g}, \mathbb{F}_{p}\right)$, especially triviality and nontriviality of them, and thereby fill the lack of knowledge of $\bmod p$ Morita-Mumford classes. Let us introduce the content of this paper briefly. In $\S 2$, we will recall relevant definitions and facts concerning mapping class groups, Morita-Mumford classes, and Harer's stability theorem. In $\S 3$, we will review results in [1], [21] concerning Morita-Mumford classes on finite subgroups of $\Gamma_{g}$. $\S 4$ is devoted to prove the following theorem which is the main result of this paper.

Theorem. If $n \equiv-1(\bmod p-1)$, then $e_{n}^{(p)} \in H^{2 n}\left(\Gamma_{g}, \mathbb{F}_{p}\right)$ is nilpotent. In particular, $e_{n}^{(2)} \in H^{2 n}\left(\Gamma_{g}, \mathbb{F}_{2}\right)$ is nilpotent for all $n \geq 0$. Conversely, if $n \not \equiv-1(\bmod p-1)$, then $e_{n}^{(p)} \in H^{2 n}\left(\Gamma_{\bullet}, \mathbb{F}_{p}\right)$ is nontrivial and is not nilpotent.

Here $H^{*}\left(\Gamma_{\bullet}, \mathbb{F}_{p}\right)=\lim _{g \rightarrow \infty} H^{*}\left(\Gamma_{g}, \mathbb{F}_{p}\right)$ is the stable mod $p$ cohomology of mapping class groups which exists by virtue of Harer's stability theorem and $e_{n}^{(p)} \in H^{2 n}\left(\Gamma_{\bullet}, \mathbb{F}_{p}\right)$ is the stable $\bmod p$ Morita-Mumford class (see $\S 2$ ). In view of the theorem, it is reasonable to make the following conjecture.

Conjecture 1. If $n \equiv-1(\bmod p-1)$, then $e_{n}^{(p)} \in H^{2 n}\left(\Gamma_{g}, \mathbb{F}_{p}\right)$ vanishes.

Passing to the stable mod $p$ cohomology, Conjecture 1 together with the prescribed theorem implies the following conjecture.

Conjecture 2. The stable mod $p$ Morita-Mumford class $e_{n}^{(p)} \in$ $H^{2 n}\left(\Gamma_{\bullet}, \mathbb{F}_{p}\right)$ vanishes if and only if $n \equiv-1(\bmod p-1)$. 
In $\S 5$ and $\S 6$, we will give a few pieces of evidence which support the conjectures. In particular, Conjecture 1 is settled in the affirmative for the following cases: (i) $n=1$, (ii) $g=2$, (iii) $g=3$ and $p=2$, (iv) $g=(p-1) / 2$ and $n \geq p-3$.

In $\S 7$, we will deal with oriented $\Sigma_{g}$-bundles. Any oriented $\Sigma_{g}$-bundle $E \rightarrow X$ is determined by the holonomy homomorphism $h: \pi_{1}(X) \rightarrow \Gamma_{g}$, and its $\bmod p$ Morita-Mumford classes $e_{n}^{(p)} \in H^{2 n}\left(X, \mathbb{F}_{p}\right)$ are nothing but the pull-back of $e_{n}^{(p)} \in H^{2 n}\left(\Gamma_{g}, \mathbb{F}_{p}\right)$ by $h$. In the context of oriented $\Sigma_{g^{-}}$ bundles, there is yet another affirmative evidence for the conjectures. In particular, we will prove the following result which is a consequence of the Grothendieck Riemann-Roch theorem.

THEOREM. Let $p$ be a prime and $E \rightarrow X$ an oriented $\Sigma_{g}$-bundle over a closed oriented $2 n$-manifold $X$. If $n$ is odd and satisfies $n \equiv-1(\bmod p-1)$, then $e_{n}^{(p)}=0$ in $H^{2 n}\left(X, \mathbb{F}_{p}\right)$.

The last section is devoted to applications. For any oriented $\Sigma_{g}$-bundle $E \rightarrow \Sigma_{h}$ over a closed oriented surface $\Sigma_{h}$, the total space $E$ is oriented nullcobordant if and only if the signature $\sigma(E)$ of $E$ vanishes (see Remark 6). Meyer [26] showed that $\sigma(E)=0$ whenever $g=2$, and showed that for every $g \geq 3$ there exists an integer $h \geq 0$ and an oriented $\Sigma_{g}$-bundle $E \rightarrow \Sigma_{h}$ with $\sigma(E) \neq 0$. Together with results of Igusa [18] and Faber [8], the vanishing of mod 2 Morita-Mumford classes of $\Gamma_{2}$ and $\Gamma_{3}$ which will be proved in $\S 6$ implies the following result which extends results of Meyer for $g=2$ and 3 .

TheOREm. Let $E \rightarrow X$ be an oriented $\Sigma_{g}$-bundle over a closed oriented manifold $X$. (i) If $g=2$, then $E$ is oriented null-cobordant. (ii) If $g=3, \operatorname{dim} X \geq 3$, and all the rational Pontryagin classes of $X$ vanish, then E is oriented null-cobordant.

Notation. Given a prime $p$, the field with $p$ elements is denoted by $\mathbb{F}_{p}$. Given a group $G$ and its subgroup $H$, the restriction is denoted by $\operatorname{res}_{H}^{G}$ : $H^{*}(G, \mathbb{Z}) \rightarrow H^{*}(H, \mathbb{Z})$. When $H$ is of finite index in $G$, the transfer (or the corestriction in the literature) is denoted by $\operatorname{Tr}_{H}^{G}: H^{*}(H, \mathbb{Z}) \rightarrow H^{*}(G, \mathbb{Z})$. Given an element $\gamma \in G$, the cyclic subgroup generated by $\gamma$ is denoted by $\langle\gamma\rangle$. Given a closed manifold $X$, its mod 2 fundamental class is denoted by $[X]_{2}$. When $X$ is oriented, its fundamental class is denoted by $[X]$. 


\section{§2. Review of mapping class groups and Morita-Mumford classes}

Let $\Sigma_{g}$ be a closed oriented surface of genus $g \geq 2$. Let Diff $+\Sigma_{g}$ be the group of orientation-preserving diffeomorphisms of $\Sigma_{g}$ equipped with $C^{\infty}$-topology. The mapping class group $\Gamma_{g}$ of $\Sigma_{g}$ is defined to be the group of connected components of Diff $+\Sigma_{g}$ :

$$
\Gamma_{g}:=\pi_{0}\left(\operatorname{Diff}_{+} \Sigma_{g}\right) .
$$

Let Diff $+\left(\Sigma_{g}, *\right)$ be the subgroup of Diff $+\Sigma_{g}$ consisting of all the orientationpreserving diffeomorphisms which fix the distinguished base point $* \in \Sigma_{g}$. The group

$$
\Gamma_{g}^{1}:=\pi_{0}\left(\operatorname{Diff}_{+}\left(\Sigma_{g}, *\right)\right)
$$

is called the mapping class group of $\Sigma_{g}$ relative to the base point $* \in \Sigma_{g}$. The natural homomorphism $\pi: \Gamma_{g}^{1} \rightarrow \Gamma_{g}$ gives rise to an extension

$$
1 \longrightarrow \pi_{1}\left(\Sigma_{g}, *\right) \longrightarrow \Gamma_{g}^{1} \stackrel{\pi}{\longrightarrow} \Gamma_{g} \longrightarrow 1 .
$$

Let $D \subset \Sigma_{g}$ be the fixed embedded disk and write $\Sigma_{g, 1}=\Sigma_{g} \backslash \operatorname{Int} D$. Let $\operatorname{Diff}_{+}\left(\Sigma_{g, 1}, \partial \Sigma_{g, 1}\right)$ be the group of all the orientation-preserving diffeomorphisms of $\Sigma_{g, 1}$ which fix the boundary $\partial \Sigma_{g, 1}$ pointwise. The group

$$
\Gamma_{g, 1}:=\pi_{0}\left(\operatorname{Diff}_{+}\left(\Sigma_{g, 1}, \partial \Sigma_{g, 1}\right)\right)
$$

is called the mapping class group of $\Sigma_{g, 1}$ relative to the one boundary component. The natural homomorphism $\Gamma_{g, 1} \rightarrow \Gamma_{g}^{1}$ gives rise to a central extension

$$
0 \longrightarrow \mathbb{Z} \longrightarrow \Gamma_{g, 1} \longrightarrow \Gamma_{g}^{1} \longrightarrow 1
$$

where the central subgroup $\mathbb{Z}$ is generated by the Dehn twist along a separating simple closed curve parallel to the boundary $\partial \Sigma_{g, 1}$.

The inclusion $\Sigma_{g, 1} \hookrightarrow \Sigma_{g+1,1}$ induces a homomorphism $\Gamma_{g, 1} \rightarrow \Gamma_{g+1,1}$. Harer's stability theorem [13] as improved by Ivanov [19] asserts that homomorphisms $\Gamma_{g, 1} \rightarrow \Gamma_{g+1,1}$ and $\Gamma_{g, 1} \rightarrow \Gamma_{g}$ induce isomorphisms

$$
H_{n}\left(\Gamma_{g, 1}, \mathbb{Z}\right) \stackrel{\cong}{\longrightarrow} H_{n}\left(\Gamma_{g+1,1}, \mathbb{Z}\right) \text { and } H_{n}\left(\Gamma_{g, 1}, \mathbb{Z}\right) \stackrel{\cong}{\longrightarrow} H_{n}\left(\Gamma_{g}, \mathbb{Z}\right)
$$

when $n<g / 2$. Hence we may define the stable cohomology of mapping class groups by

$$
H^{*}\left(\Gamma_{\bullet}, \mathbb{Z}\right):=\lim _{g \rightarrow \infty} H^{*}\left(\Gamma_{g, 1}, \mathbb{Z}\right)
$$


Let $e \in H^{2}\left(\Gamma_{g}^{1}, \mathbb{Z}\right)$ be the Euler class of the central extension (2.1). The $n$-th Morita-Mumford class $e_{n} \in H^{2 n}\left(\Gamma_{g}, \mathbb{Z}\right)(n \geq 0)$ is then defined by

$$
e_{n}=\pi_{!}\left(e^{n+1}\right) \in H^{2 n}\left(\Gamma_{g}, \mathbb{Z}\right),
$$

where $\pi_{!}: H^{*}\left(\Gamma_{g}^{1}, \mathbb{Z}\right) \rightarrow H^{*-2}\left(\Gamma_{g}, \mathbb{Z}\right)$ is the Gysin homomorphism. Note that $e_{0}=\chi\left(\Sigma_{g}\right)=2-2 g$. Given a prime number $p$, the $\bmod p$ MoritaMumford class $e_{n}^{(p)} \in H^{*}\left(\Gamma_{g}, \mathbb{F}_{p}\right)$ is defined to be the $\bmod p$ reduction of $e_{n} \in H^{*}\left(\Gamma_{g}, \mathbb{Z}\right)$. By the naturality of the Gysin homomorphism with respect to the $\bmod p$ reduction, one has

$$
e_{n}^{(p)}=\pi_{!}\left(\left(e^{(p)}\right)^{n+1}\right) \in H^{*}\left(\Gamma_{g}, \mathbb{F}_{p}\right),
$$

where $e^{(p)} \in H^{2}\left(\Gamma_{g}^{1}, \mathbb{F}_{p}\right)$ is the $\bmod p$ reduction of $e \in H^{2}\left(\Gamma_{g}^{1}, \mathbb{Z}\right)$ which is referred as the mod $p$ Euler class of the central extension (2.1).

Let $e_{n} \in H^{2 n}\left(\Gamma_{g, 1}, \mathbb{Z}\right)$ be the image of $e_{n} \in H^{2 n}\left(\Gamma_{g}, \mathbb{Z}\right)$ under the homomorphism induced by $\Gamma_{g, 1} \rightarrow \Gamma_{g}$ (we use the same symbol). As was shown by Miller [27] and Morita [28], $e_{n}$ is preserved by the homomorphisms induced by $\Gamma_{g, 1} \rightarrow \Gamma_{g+1,1}$ and $\Gamma_{g, 1} \rightarrow \Gamma_{g}$. Hence the stable Morita-Mumford classes $e_{n} \in H^{2 n}\left(\Gamma_{\bullet}, \mathbb{Z}\right)$ make sense. We close this section by proving nontriviality of mod $p$ Euler class $e^{(p)} \in H^{2}\left(\Gamma_{g}^{1}, \mathbb{F}_{p}\right)$ along the lines of Harer $[16$, Theorem 7.1] and Morita [28, p. 559].

Proposition 1. For any prime $p$, the mod $p$ Euler class $e^{(p)} \in$ $H^{2}\left(\Gamma_{g}^{1}, \mathbb{F}_{p}\right)$ of the central extension (2.1) is nontrivial if $g$ is sufficiently large.

Proof. Since $\Gamma_{g, 1} \rightarrow \Gamma_{g}$ is the composition of the homomorphisms $\Gamma_{g, 1} \rightarrow \Gamma_{g}^{1}$ and $\Gamma_{g}^{1} \rightarrow \Gamma_{g}$, Harer's stability theorem implies that the induced homomorphism $H^{n}\left(\Gamma_{g}^{1}, \mathbb{F}_{p}\right) \rightarrow H^{n}\left(\Gamma_{g, 1}, \mathbb{F}_{p}\right)$ is surjective for $n<g / 2$. This shows that the Gysin sequence

$$
\cdots \rightarrow H^{n-2}\left(\Gamma_{g}^{1}, \mathbb{F}_{p}\right) \stackrel{\cup e^{(p)}}{\rightarrow} H^{n}\left(\Gamma_{g}^{1}, \mathbb{F}_{p}\right) \rightarrow H^{n}\left(\Gamma_{g, 1}, \mathbb{F}_{p}\right) \rightarrow H^{n}\left(\Gamma_{g}^{1}, \mathbb{F}_{p}\right) \rightarrow \cdots
$$

splits into the short exact sequence

$$
0 \longrightarrow H^{n-2}\left(\Gamma_{g}^{1}, \mathbb{F}_{p}\right) \stackrel{\cup e^{(p)}}{\longrightarrow} H^{n}\left(\Gamma_{g}^{1}, \mathbb{F}_{p}\right) \longrightarrow H^{n}\left(\Gamma_{g, 1}, \mathbb{F}_{p}\right) \longrightarrow 0
$$

for $n<g / 2$ and the proposition follows.

The last proposition suggests the notriviality of the conjectures stated in the introduction.

Remark 1. When $p \geq 5$, the $\bmod p$ Euler class $e^{(p)} \in H^{2}\left(\Gamma_{g}^{1}, \mathbb{F}_{p}\right)$ is actually nontrivial for all $g \geq 3$. See Corollary 2 . 


\section{§3. Morita-Mumford classes on finite subgroups}

Let $G$ be a finite subgroup of $\Gamma_{g}$. By the affirmative solution of the Nielsen realization problem due to Kerckhoff [22], $G$ is realized as a group of automorphisms of a suitable compact Riemann surface $R$ of genus $g$. For each $x \in R$, let $G_{x}$ be the isotropy subgroup at $x$. Since $G$ preserves the orientation, $G_{x}$ is necessarily cyclic. Set $\mathcal{S}=\left\{x \in R: G_{x} \neq 1\right\}$, and let $\mathcal{S} / G$ be a set of representatives of $G$-orbits of elements of $\mathcal{S}$. For each $x \in \mathcal{S} / G$, let $\xi_{x}$ be the flat complex line bundle over the classifying space $B G_{x}$ of $G_{x}$ associated to the action of $G_{x}$ on the holomorphic tangent space $T_{x} R$, and let $c_{1}\left(\xi_{x}\right) \in H^{2}\left(G_{x}, \mathbb{Z}\right)$ be its first Chern class. Among other things, Kawazumi and Uemura proved the following result.

THEOREM 1. ([21]) In the situation stated above, one has

$$
\operatorname{res}_{G}^{\Gamma_{g}} e_{n}=\sum_{x \in \mathcal{S} / G} \operatorname{Tr}_{G_{x}}^{G}\left(c_{1}\left(\xi_{x}\right)^{n}\right) \in H^{2 n}(G, \mathbb{Z})
$$

for all $n \geq 0$.

Now we deal with finite cyclic subgroups of $\Gamma_{g}$. Let $\gamma \in \Gamma_{g}$ be an element of order $m$. As before, choose a compact Riemann surface $R$ of genus $g$ for which $\langle\gamma\rangle$ is a group of automorphisms. Let $\left\{x_{i}\right\}_{1 \leq i \leq q}$ be a set of representatives of the singular orbits of $\langle\gamma\rangle$, and $\alpha_{i}$ the order of the isotropy subgroup at $x_{i}$. Let $\beta_{i}$ be an integer such that $\gamma^{\beta_{i} m / \alpha_{i}}$ acts on $T_{x_{i}} R$ by $z \mapsto \exp \left(2 \pi \sqrt{-1} / \alpha_{i}\right) z$ with respect to a suitable local coordinate $z$ at $x_{i}$. The number $\beta_{i}$ is well-defined modulo $\alpha_{i}$ and is prime to $\alpha_{i}$. The fixed point data of $\gamma$ is then the collection

$$
\left\langle g, m \mid \beta_{1} / \alpha_{1}, \ldots, \beta_{q} / \alpha_{q}\right\rangle .
$$

The rational numbers $\beta_{1} / \alpha_{1}, \ldots, \beta_{q} / \alpha_{q}$ are unique up to order, if we consider them as elements in $\mathbb{Q} / \mathbb{Z}$. The fixed point data satisfies

$$
\sum_{i=1}^{q} \frac{\beta_{i}}{\alpha_{i}} \in \mathbb{Z}
$$

and the Riemann-Hurwitz equation

$$
2 g-2=m(2 h-2)+m \sum_{i=1}^{q}\left(1-\frac{1}{\alpha_{i}}\right)
$$


for some integer $h \geq 0$. According to Symonds [37], the fixed point data is independent of various choices made and hence is well defined for $\gamma \in \Gamma_{g}$. For later use, we recall the following fact concerning a realization of fixed point data.

Proposition 2. Let $p$ be a prime and $\beta_{1}, \ldots, \beta_{q}$ integers prime to $p$. Then $\left\langle g, p \mid \beta_{1} / p, \ldots, \beta_{q} / p\right\rangle$ can be realized as the fixed point data of an element of $\Gamma_{g}$ of order $p$ if and only if it satisfies (3.2) and (3.3).

Proof. See [10].

Now let $\gamma \in \Gamma_{g}$ be an element of order $m$ having the fixed point data $\left\langle g, m \mid \beta_{1} / \alpha_{1}, \ldots, \beta_{q} / \alpha_{q}\right\rangle$ as before. Let $\xi$ be the complex line bundle over the classifying space $B\langle\gamma\rangle$ of $\langle\gamma\rangle$ associated to the representation $\langle\gamma\rangle \rightarrow$ $U(1)$ defined by $\gamma \mapsto \exp (2 \pi i / m)$, and let $u_{\gamma} \in H^{2}(\langle\gamma\rangle, \mathbb{Z})$ be the first Chern class of $\xi$. As is known, one has

$$
H^{*}(\langle\gamma\rangle, \mathbb{Z}) \cong \mathbb{Z}\left[u_{\gamma}\right] /\left(m u_{\gamma}\right)
$$

Under these conventions, Theorem 1 reduces to the following result.

Proposition 3. Let $\gamma \in \Gamma_{g}$ be an element of order $m$ having the fixed point data $\left\langle g, m \mid \beta_{1} / \alpha_{1}, \ldots, \beta_{q} / \alpha_{q}\right\rangle$. Then

$$
\operatorname{res}_{\langle\gamma\rangle}^{\Gamma_{g}} e_{n}=\sum_{i=1}^{q} \frac{m}{\alpha_{i}}\left(\beta_{i}^{*}\right)^{n} u_{\gamma}^{n} \in H^{2 n}(\langle\gamma\rangle, \mathbb{Z}),
$$

where $\beta_{i}^{*}$ is an integer satisfying $\beta_{i}^{*} \beta_{i} \equiv 1\left(\bmod \alpha_{i}\right)$.

Proof. See [1].

Proposition 4. Let $G$ be a finite subgroup of $\Gamma_{g}$ whose Sylow $p$ subgroup is cyclic. Then

$$
\operatorname{res}_{G}^{\Gamma_{g}} e_{n}=0 \text { in } H^{2 n}\left(G, \mathbb{F}_{p}\right)
$$

holds for all $n \equiv-1(\bmod p-1)$. 
Proof. Let $G_{p}$ be a Sylow $p$-subgroup of $G$ generated by an element $\gamma \in G_{p}$ having the fixed point data

$$
\left\langle g, p^{t} \mid \beta_{1} / p^{t_{1}}, \ldots, \beta_{q} / p^{t_{q}}\right\rangle .
$$

Choose an integer $\beta_{i}^{*}$ satisfying $\beta_{i} \beta_{i}^{*} \equiv 1\left(\bmod p^{t_{i}}\right)$. Since $\left(\beta_{i}^{*}\right)^{p-1} \equiv 1$ $(\bmod p)$, we see that, if $n \equiv-1(\bmod p-1)$ then $\left(\beta_{i}^{*}\right)^{n} \equiv \beta_{i}(\bmod p)$. On the other hand, it follows from (3.2) that

$$
\sum_{i=1}^{q} p^{t-t_{i}} \beta_{i} \equiv 0 \quad(\bmod p)
$$

Applying Proposition 3 , we see that, if $n \equiv-1(\bmod p-1)$ then

$$
\operatorname{res}_{G_{p}}^{\Gamma_{g}} e_{n}=\sum_{i=1}^{q} p^{t-t_{i}}\left(\beta_{i}^{*}\right)^{n} u_{\gamma}^{n}=\sum_{i=1}^{q} p^{t-t_{i}} \beta_{i} u_{\gamma}^{n}=0
$$

in $H^{2 n}\left(G_{p}, \mathbb{F}_{p}\right)$. As $\operatorname{res}_{G_{p}}^{G}: H^{*}\left(G, \mathbb{F}_{p}\right) \rightarrow H^{*}\left(G_{p}, \mathbb{F}_{p}\right)$ is injective, this completes the proof.

Remark 2. It was proved in [1] that, for any cyclic subgroup $C \subset \Gamma_{g}$ of order $m, \operatorname{res}_{C}^{\Gamma_{g}} e_{n} \in H^{2 n}(C, \mathbb{Z})$ vanishes whenever $n \equiv-1 \bmod \phi(m)$, where $\phi$ is the Euler function.

\section{$\S 4$. Proof of the main result}

The purpose of this section is to prove the main result of this paper which was mentioned in the introduction. More precisely, we will prove the following two theorems.

THEOREM 2. If $n \equiv-1(\bmod p-1)$, then $e_{n}^{(p)} \in H^{2 n}\left(\Gamma_{g}, \mathbb{F}_{p}\right)$ is nilpotent. In particular, $e_{n}^{(2)} \in H^{2 n}\left(\Gamma_{g}, \mathbb{F}_{2}\right)$ is nilpotent for all $n \geq 0$.

THEOREM 3. If $n \not \equiv-1(\bmod p-1)$, then $\left(e_{n}^{(p)}\right)^{k} \in H^{2 n k}\left(\Gamma_{g}, \mathbb{F}_{p}\right)$ is nontrivial for any $k \geq 1$ satisfying $k<g / 4 n$.

Passing to the stable mod $p$ cohomology $H^{*}\left(\Gamma_{\bullet}, \mathbb{F}_{p}\right)$, Theorem 3 together with Harer's stability theorem implies the following result which was mentioned in the introduction. 
Corollary 1. The stable mod $p$ Morita-Mumford class $e_{n}^{(p)} \in$ $H^{2 n}\left(\Gamma_{\bullet}, \mathbb{F}_{p}\right)$ is nontrivial and is not nilpotent whenever $n \not \equiv-1(\bmod p-1)$.

Let $p$ be a prime number. Recall that a finite group $E$ is called an elementary abelian p-group of rank $m$ if $E \cong(\mathbb{Z} / p \mathbb{Z})^{m}$. Let us denote $\operatorname{rank}_{p} E=m$. We begin with the following lemma which may be wellknown.

LEMMA. Let $p$ be a prime number and $E$ an elementary abelian $p$ group with $\operatorname{rank}_{p} E \geq 2$. For any cyclic subgroup $C \subset E$ of order $p$, the transfer

$$
\operatorname{Tr}_{C}^{E}: H^{*}(C, \mathbb{Z}) \longrightarrow H^{*}(E, \mathbb{Z})
$$

is the zero homomorphism for $*>0$.

Proof. It is easy to see that the restriction $\operatorname{res}_{C}^{E}: H^{2}(E, \mathbb{Z}) \rightarrow H^{2}(C, \mathbb{Z})$ is surjective. Since $H^{*}(C, \mathbb{Z}) \cong \mathbb{Z}[x] /(p x)$ with $\operatorname{deg} x=2$, it follows that $\operatorname{res}_{C}^{E}: H^{*}(E, \mathbb{Z}) \rightarrow H^{*}(C, \mathbb{Z})$ is also surjective. Given an element $u \in$ $H^{*}(C, \mathbb{Z})$, choose $v \in H^{*}(E, \mathbb{Z})$ with $u=\operatorname{res}_{C}^{E} v$, and one has

$$
\operatorname{Tr}_{C}^{E} u=\operatorname{Tr}_{C}^{E} \circ \operatorname{res}_{C}^{E} v=(E: C) \cdot v
$$

where $(E: C)$ is the index of $C$ in $E$. It follows that $p v=0$ since $H^{2}(E, \mathbb{Z}) \cong$ $(\mathbb{Z} / p \mathbb{Z})^{m}$ with $m=\operatorname{rank}_{p} E$ and $v$ can be chosen as a product of elements of $H^{2}(E, \mathbb{Z})$. As $p$ divides $(E: C)$, this proves the lemma.

Proposition 5. Let $p$ be a prime number. For any elementary abelian $p$-subgroups $E$ of $\Gamma_{g}$ with $\operatorname{rank}_{p} E \geq 2$, one has

$$
\operatorname{res}_{E}^{\Gamma_{g}} e_{n}=0 \text { in } H^{2 n}(E, \mathbb{Z})
$$

for all $n \geq 1$.

Proof. According to Theorem 1, $\operatorname{res}_{E}^{\Gamma_{g}} e_{n}$ is the sum of elements each of which belongs to the image of the transfer from a cyclic subgroup of order $p$. In view of the lemma, this completes the proof.

Remark 3. Proposition 5 should be compared with a result of Morita [29] which asserts that, for every amenable subgroup $A \subset \Gamma_{g}$, one has $\operatorname{res}_{A}^{\Gamma_{g}} e_{n}=0$ in $H^{2 n}(A, \mathbb{Q})$ for all $n \geq 1$. Consequently, if $A \subset \Gamma_{g}$ is a free abelian subgroup of finite rank, then

$$
\operatorname{res}_{A}^{\Gamma_{g}} e_{n}=0 \text { in } H^{2 n}(A, \mathbb{Z})
$$

for all $n \geq 1$. 
Now we are ready to prove Theorem 2. The key ingredient for the proof of Theorem 2 is the celebrated F-isomorphism theorem of Quillen:

THEOREM 4. ([36]) Let $\Gamma$ be a group having finite virtual cohomological dimension. If $u \in H^{*}\left(\Gamma, \mathbb{F}_{p}\right)$ restricts to zero for every elementary abelian p-subgroups, then $u$ is nilpotent.

Proof of Theorem 2. Recall that the virtual cohomological dimension of $\Gamma_{g}$ (written $\operatorname{vcd} \Gamma_{g}$ ) is finite. Actually, $\operatorname{vcd} \Gamma_{g}=4 g-5$ according to Harer [14]. In view of Proposition 4 and 5, Theorem 2 follows from Theorem 4.

Proof of Theorem 3. Given an odd prime number $p$, choose a primitive root $r_{p}\left(1<r_{p} \leq p-1\right)$ which is a generator of the multiplicative group $\mathbb{F}_{p}^{\times}=\mathbb{F}_{p} \backslash\{0\}$. Choose an integer $k \geq 1$ satisfying

$$
h:=p k+\frac{(p-1)\left(p-r_{p}-1\right)}{2} \geq g
$$

Let $\gamma \in \Gamma_{h}$ be an element having the fixed point data

$$
\langle h, p \mid \underbrace{1 / p, \ldots, 1 / p}_{p-r_{p}}, r_{p} / p\rangle .
$$

Such $\gamma \in \Gamma_{h}$ exists by virtue of Proposition 2. Applying Proposition 3 to $\gamma \in \Gamma_{h}$, we see that

$$
\operatorname{res}_{\langle\gamma\rangle}^{\Gamma_{h}} e_{n}=\left(p-r_{p}+\left(r_{p}^{*}\right)^{n}\right) u_{\gamma} \text { in } H^{2 n}(\langle\gamma\rangle, \mathbb{Z})
$$

where $r_{p}^{*}$ is an integer satisfying $r_{p}^{*} r_{p} \equiv 1(\bmod p)$. Since $r_{p}$ is a primitive root, $p-r_{p}+\left(r_{p}^{*}\right)^{n} \equiv 0(\bmod p)$ if and only if $n \equiv-1(\bmod p-1)$. As

$$
H^{*}\left(\langle\gamma\rangle, \mathbb{F}_{p}\right) \cong \mathbb{F}_{p}\left[u_{\gamma}, v\right] /\left(v^{2}\right)
$$

with $\operatorname{deg} v=1$, we conclude that the $\bmod p$ Morita-Mumford class $e_{n}^{(p)} \in$ $H^{2 n}\left(\Gamma_{h}, \mathbb{F}_{p}\right)$ is nontrivial and is not nilpotent whenever $n \not \equiv-1(\bmod p-1)$. Now the theorem follows from Harer's stability theorem. 


\section{$\S 5 . \quad$ First Morita-Mumford classes}

The natural action of $\Gamma_{g}$ on the first cohomology $H^{1}\left(\Sigma_{g}, \mathbb{Z}\right)$ of $\Sigma_{g}$ preserves the symplectic form on it given by the cup product. Hence if we choose a symplectic basis for $H^{1}\left(\Sigma_{g}, \mathbb{Z}\right)$, we obtain a homomorphism

$$
\Gamma_{g} \longrightarrow S p(2 g, \mathbb{Z})
$$

where $S p(2 g, \mathbb{Z})$ is the Siegel modular group of degree $g$. This induces a homomorphism

$$
\Gamma_{g} \longrightarrow S p(2 g, \mathbb{R}) \text {. }
$$

Now the maximal compact subgroup of $S p(2 g, \mathbb{R})$ is isomorphic to $U(g)$. Hence passing to the classifying spaces we obtain a continuous map

$$
B \Gamma_{g} \longrightarrow B U(g) .
$$

Let $c_{n} \in H^{2 n}\left(\Gamma_{g}, \mathbb{Z}\right)$ be the pull-back of the universal Chern class $c_{n} \in$ $H^{2 n}(B U(g), \mathbb{Z})$. According to Morita [28] and Mumford [35], one has

$$
e_{k-1}-e_{k-2} c_{1}+\cdots+(-1)^{g} e_{k-g-1} c_{g}=0 \text { in } H^{2(k-1)}\left(\Gamma_{g}, \mathbb{Z}\right)
$$

for all $k \geq g$, where we understand $e_{-1}=0$.

Proposition 6. For all $g \geq 2$, one has $e_{1}=-12 c_{1}$ in $H^{2}\left(\Gamma_{g}, \mathbb{Z}\right)$.

Proof. Over the rationals, one has

$$
e_{1}=-12 c_{1} \text { in } H^{2}\left(\Gamma_{g}, \mathbb{Q}\right) .
$$

This can be proved by applying the Atiyah-Singer index theorem for families of elliptic operators or the Grothendieck Riemann-Roch theorem (see [28], [35] and $\S 7$ below). On the other hand, Harer [12], [15] showed that $H^{2}\left(\Gamma_{g}, \mathbb{Z}\right)$ is generated by the first Chern class $c_{1}$ and that

$$
H^{2}\left(\Gamma_{g}, \mathbb{Z}\right) \cong \begin{cases}\mathbb{Z} / 10 \mathbb{Z}, & g=2 \\ \mathbb{Z}, & g \geq 3 .\end{cases}
$$

The proposition for $g \geq 3$ follows from (5.2) and (5.3). When $g=2$, (5.1) for $k=2$ implies $e_{1}=-2 c_{1}$ in $H^{2}\left(\Gamma_{2}, \mathbb{Z}\right)$ since $e_{0}=-2$. In view of (5.3), this proves the proposition. 
Proposition 6 and the previously mentioned results of Harer imply the following corollary which is consistent with the conjectures.

Corollary 2. For all $g \geq 2, e_{1}^{(2)} \in H^{2}\left(\Gamma_{g}, \mathbb{F}_{2}\right)$ and $e_{1}^{(3)} \in H^{2}\left(\Gamma_{g}, \mathbb{F}_{3}\right)$ vanish. Furthermore, for all $p \geq 5$ and $g \geq 3, e_{1}^{(p)} \in H^{2}\left(\Gamma_{g}, \mathbb{F}_{p}\right)$ is nontrivial.

Now we prove nilpotency of first $\bmod p$ Morita-Mumford classes and thereby show that the converse of Theorem 2 does not hold.

Proposition 7. For all $g \geq 3$, the first $\bmod p$ Morita-Mumford class $e_{1}^{(p)} \in H^{2}\left(\Gamma_{g}, \mathbb{F}_{p}\right)$ is nontrivial and is nilpotent whenever $p>4 g+2$.

Proof. If $\Gamma_{g}$ has no elements of order $p$, then $H^{n}\left(\Gamma_{g}, \mathbb{F}_{p}\right)$ vanishes for all $n>\operatorname{vcd} \Gamma_{g}=4 g-5$. This can be proved by inspection of the FarrellTate cohomology of $\Gamma_{g}$ (see [4] for Farrell-Tate cohomology). On the other hand, as was proved by Wiman a hundred years ago (see [17]), if $p>4 g+2$ then $\Gamma_{g}$ has no elements of order $p$. In summary, we have

$$
H^{n}\left(\Gamma_{g}, \mathbb{F}_{p}\right)=0 \quad \text { if } p>4 g+2 \text { and } n>4 g-5 \text {. }
$$

Together with Corollary 2, this completes the proof.

As for higher mod $p$ Morita-Mumford classes, Theorem 3 and (5.4) imply the following result.

Proposition 8. If $p>4 g+2$, then $e_{n}^{(p)} \in H^{2 n}\left(\Gamma_{g}, \mathbb{F}_{p}\right)$ is nontrivial and is nilpotent for all $n<g / 4$ with $n \not \equiv-1(\bmod p-1)$.

\section{$\S 6 . \quad$ Various calculations}

The purpose of this section is to give a few pieces of evidence which support the conjectures (Propositions 9, 10 and 11).

Proposition 9. For $g=2$ or 3, the mod 2 Morita-Mumford class $e_{n}^{(2)} \in H^{2 n}\left(\Gamma_{g}, \mathbb{F}_{2}\right)$ vanishes for all $n \geq 0$.

Proof. Applying the mod 2 reduction to (5.1), we obtain

$$
e_{k-1}^{(2)}+e_{k-2}^{(2)} w_{2}+\cdots+e_{k-g-1}^{(2)} w_{2 g}=0 \text { in } H^{2(k-1)}\left(\Gamma_{g}, \mathbb{F}_{2}\right)
$$


for all $k \geq g$, where $w_{2 i} \in H^{2 i}\left(\Gamma_{g}, \mathbb{F}_{2}\right)$ is the pull-back of the symplectic Stiefel-Whitney class $w_{2 i} \in H^{2 i}\left(B S p(2 g, \mathbb{R}), \mathbb{F}_{2}\right)$ by $\Gamma_{g} \rightarrow S p(2 g, \mathbb{R})$. When $g=2$, we have

$$
e_{k-1}^{(2)}+e_{k-2}^{(2)} w_{2}+e_{k-3}^{(2)} w_{4}=0 \text { in } H^{2(k-1)}\left(\Gamma_{2}, \mathbb{F}_{2}\right)
$$

for all $k \geq 2$. Since $e_{1}^{(2)}=e_{0}^{(2)}=0$ by Corollary 2, the iterated use of $(6.2)$ implies the proposition for $g=2$. When $g=3$, we have

$$
e_{k-1}^{(2)}+e_{k-2}^{(2)} w_{2}+e_{k-3}^{(2)} w_{4}+e_{k-4}^{(2)} w_{6}=0 \text { in } H^{2(k-1)}\left(\Gamma_{3}, \mathbb{F}_{2}\right)
$$

for all $k \geq 3$. Applying $k=3$ to (6.3), we see that $e_{2}^{(2)}$ vanishes by Corollary 2. Hence we have $e_{2}^{(2)}=e_{1}^{(2)}=e_{0}^{(2)}=0$. By the iterated use of (6.3), the proposition for $g=3$ is proved.

Proposition 10. For $p=3$ or 5 , the $\bmod p$ Morita-Mumford class $e_{n}^{(p)} \in H^{2 n}\left(\Gamma_{2}, \mathbb{F}_{p}\right)$ of $\Gamma_{2}$ vanishes if and only if $n \equiv-1(\bmod p-1)$. Furthermore, if $n \not \equiv-1(\bmod p-1)$ then $e_{n}^{(p)} \in H^{2 n}\left(\Gamma_{2}, \mathbb{F}_{p}\right)$ is not nilpotent.

Proof. We have

$$
e_{k-1}^{(3)}+e_{k-2}^{(3)} c_{1}+e_{k-3}^{(3)} c_{2}=0 \text { in } H^{2(k-1)}\left(\Gamma_{2}, \mathbb{F}_{3}\right)
$$

for all $k \geq 2$. Here the $\bmod 3$ reduction of $c_{i} \in H^{2 i}\left(\Gamma_{3}, \mathbb{Z}\right)$ is denoted by the same symbol. Since $10 e_{1}^{(3)}=10 c_{1}=0$ by $(5.3)$, we have $e_{1}^{(3)}=c_{1}=0$ in $H^{*}\left(\Gamma_{2}, \mathbb{F}_{3}\right)$ and hence

$$
e_{k-1}^{(3)}=-e_{k-3}^{(3)} c_{2} \text { in } H^{2(k-1)}\left(\Gamma_{2}, \mathbb{F}_{3}\right)
$$

for all $k \geq 2$. By the iterated use of (6.4), we conclude that $e_{n}^{(3)} \in$ $H^{2 n}\left(\Gamma_{2}, \mathbb{F}_{3}\right)$ vanishes for every odd integer $n \geq 1$.

Conversely, let $\gamma \in \Gamma_{2}$ be an element of order 3 having the fixed point data $\langle 2,3 \mid 1 / 3,1 / 3,2 / 3,2 / 3\rangle$. We have

$$
\operatorname{res}_{\langle\gamma\rangle}^{\Gamma_{2}} e_{n}=\left(1+1+2^{n}+2^{n}\right) u_{\gamma}=2\left(1+2^{n}\right) u_{\gamma} \text { in } H^{*}(\langle\gamma\rangle, \mathbb{Z}) .
$$

As in the proof of Theorem 3, it follows that $e_{n}^{(3)} \in H^{2 n}\left(\Gamma_{2}, \mathbb{F}_{3}\right)$ is nontrivial and is not nilpotent for every even integer $n>0$, which verifies the proposition for $p=3$. 
Finally, we will prove the argument for $p=5$. Let $\gamma \in \Gamma_{2}$ be an element of order 5 having the fixed point data $\langle 2,5 \mid 1 / 5,1 / 5,3 / 5\rangle$. We have

$$
\operatorname{res}_{\langle\gamma\rangle}^{\Gamma_{2}} e_{n}=\left(1+1+2^{n}\right) u_{\gamma}=2\left(1+2^{n-1}\right) u_{\gamma} \text { in } H^{*}(\langle\gamma\rangle, \mathbb{Z}) .
$$

Hence $e_{n}^{(5)} \in H^{*}\left(\Gamma_{2}, \mathbb{F}_{5}\right)$ is nontrivial and is not nilpotent if $n \not \equiv-1$ ( $\bmod 4$ ). According to Cohen [5] (see also [20]), there is a cyclic subgroup $C \subset \Gamma_{2}$ of order 5 such that the inclusion $C \hookrightarrow \Gamma_{2}$ induces the isomorphism $\operatorname{res}_{C}^{\Gamma_{2}}: H^{*}\left(\Gamma_{2}, \mathbb{F}_{5}\right) \cong H^{*}\left(C, \mathbb{F}_{5}\right)$ of the $\bmod 5$ cohomology. In view of Proposition 4 , we see that $e_{n}^{(5)} \in H^{*}\left(\Gamma_{2}, \mathbb{F}_{5}\right)$ vanishes when $n \equiv-1(\bmod 4)$, which proves the proposition for $p=5$.

Remark 4. Actually, the subgroup $C \subset \Gamma_{2}$ which appeared in the last paragraph is generated by an element of $\Gamma_{2}$ having the fixed point data $\langle 2,5 \mid 1 / 5,1 / 5,3 / 5\rangle$. See [20].

Remark 5. According to a result of Lee and Weintraub [24], the mod $p$ cohomology of $\Gamma_{2}$ is nontrivial if and only if $p=2,3$, or 5 . Hence all the nontrivial mod $p$ Morita-Mumford classes of $\Gamma_{2}$ are determined by the last two propositions.

Proposition 11. Let $p$ be a prime. Then $e_{n}^{(p)} \in H^{2 n}\left(\Gamma_{(p-1) / 2}, \mathbb{F}_{p}\right)$ vanishes whenever $n \geq p-3$ and $n \equiv-1(\bmod p-1)$.

Proof. Let $\mathcal{C}_{p}$ be the set of representatives of conjugacy classes of cyclic subgroups of order $p$ of $\Gamma_{(p-1) / 2}$. According to results of Xia [39], the natural homomorphism

$$
\prod_{C \in \mathcal{C}_{p}} \operatorname{res}_{C}^{\Gamma_{(p-1) / 2}}: \widehat{H}^{n}\left(\Gamma_{(p-1) / 2}, \mathbb{Z}\right)_{(p)} \longrightarrow \prod_{C \in \mathcal{C}_{p}} \widehat{H}^{n}(C, \mathbb{Z})
$$

is injective, where $\widehat{H}^{n}(-, \mathbb{Z})$ is the Farrell-Tate cohomology and $\widehat{H}^{n}(-, \mathbb{Z})_{(p)}$ is the $p$-primary component of $\widehat{H}^{n}(-, \mathbb{Z})$. Since $\widehat{H}^{n}\left(\Gamma_{(p-1) / 2}, \mathbb{Z}\right)$ is a torsion group, the $\bmod p$ reduction $\widehat{H}^{n}\left(\Gamma_{(p-1) / 2}, \mathbb{Z}\right) \rightarrow \widehat{H}^{n}\left(\Gamma_{(p-1) / 2}, \mathbb{F}_{p}\right)$ factors through $\widehat{H}^{n}\left(\Gamma_{(p-1) / 2}, \mathbb{Z}\right)_{(p)}($ cf. [4, p. 290]). Consequently,

$$
\prod_{C \in \mathcal{C}_{p}} \operatorname{res}_{C}^{\Gamma_{(p-1) / 2}}: \widehat{H}^{n}\left(\Gamma_{(p-1) / 2}, \mathbb{F}_{p}\right) \longrightarrow \prod_{C \in \mathcal{C}_{p}} \widehat{H}^{n}\left(C, \mathbb{F}_{p}\right)
$$


is injective on the image of $\widehat{H}^{n}\left(\Gamma_{(p-1) / 2}, \mathbb{Z}\right) \rightarrow \widehat{H}^{n}\left(\Gamma_{(p-1) / 2}, \mathbb{F}_{p}\right)$, because the $\bmod p$ reduction $\widehat{H}^{*}(C, \mathbb{Z}) \rightarrow \widehat{H}^{*}\left(C, \mathbb{F}_{p}\right)$ is injective as it is easily seen. According to fundamental properties of Farrell-Tate cohomology, there is a commutative diagram

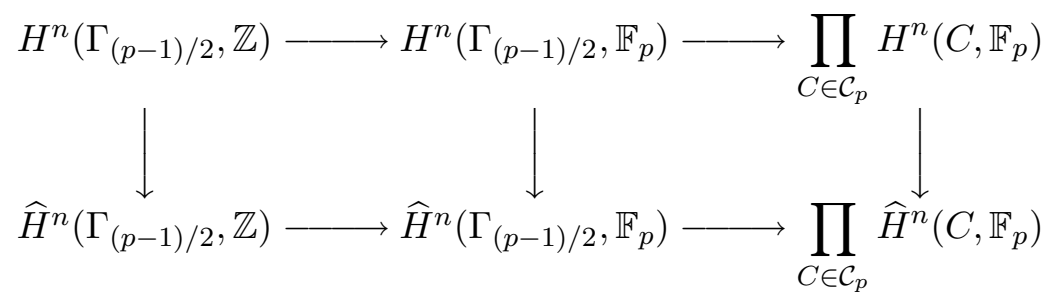

in which all the vertical arrows are isomorphisms for $n>\operatorname{vcd} \Gamma_{(p-1) / 2}=$ $2 p-7$ (cf. [4, p. 278]). In view of Proposition 4, this completes the proof.

\section{$\S 7$. Morita-Mumford classes for $\Sigma_{g}$-bundles}

In this section, we consider mod $p$ Morita-Mumford classes in the context of oriented $\Sigma_{g}$-bundles and thereby obtain affirmative evidence for Conjecture 1. A smooth fiber bundle $\pi: E \rightarrow X$ with fiber $\Sigma_{g}$ is called a $\Sigma_{g}$-bundle. Let $T_{E / X}$ be the relative tangent bundle (or the tangent bundle along the fiber) of $\pi$. Namely it is the subbundle of the tangent bundle of $E$ consisting of those vectors which are tangent to the fibers of the bundle. If $T_{E / X}$ is orientable and an orientation is given on it, we say that the $\Sigma_{g^{-}}$ bundle $\pi: E \rightarrow X$ is oriented. Given an oriented $\Sigma_{g}$-bundle $\pi: E \rightarrow X$, let

$$
e=e\left(T_{E / X}\right) \in H^{2}(E, \mathbb{Z})
$$

be the Euler class of $T_{E / X}$. The $n$-th Morita-Mumford class $e_{n} \in H^{2 n}(X, \mathbb{Z})$ of $\pi$ is then defined by

$$
e_{n}=\pi_{!}\left(e^{n+1}\right) \in H^{2 n}(X, \mathbb{Z}),
$$

where $\pi_{!}: H^{*}(E, \mathbb{Z}) \rightarrow H^{*-2}(X, \mathbb{Z})$ is the Gysin homomorphism. As is known,

$$
\pi_{!}\left(x \cup \pi^{*}(y)\right)=\pi_{!}(x) \cup y
$$

holds for any $x \in H^{*}(E, \mathbb{Z})$ and $y \in H^{*}(X, \mathbb{Z})$. According to a result of Earle and Eells [6], the classifying space $B$ Diff $_{+} \Sigma_{g}$ of oriented $\Sigma_{g}$-bundles is the Eilenberg-MacLane space $K\left(\Gamma_{g}, 1\right)$ of $\Gamma_{g}$. It follows that the isomorphism 
class of an oriented $\Sigma_{g}$-bundle $\pi: E \rightarrow X$ is completely determined by the holonomy homomorphism

$$
h: \pi_{1}(X) \longrightarrow \Gamma_{g}
$$

induced by the classifying map $X \rightarrow K\left(\Gamma_{g}, 1\right)$. In addition, the MoritaMumford classes $e_{n} \in H^{2 n}(X, \mathbb{Z})$ of $\pi$ are identified with the pull-back of $e_{n} \in H^{2 n}\left(\Gamma_{g}, \mathbb{Z}\right)$ by the holonomy homomorphism. See [28] for further detail. We will prove the following theorem.

THEOREM 5. Let $p$ be a prime, $n_{1}, n_{2}, \ldots, n_{k}$ positive integers, and $n=\sum_{i} n_{i}$. Let $E \rightarrow X$ be an oriented $\Sigma_{g}$-bundle over a closed oriented manifold $X$ whose $2 n$-th cohomology group $H^{2 n}(X, \mathbb{Z})$ is torsion-free. If $n_{i}$ is odd and satisfies $n_{i} \equiv-1(\bmod p-1)$ for some $i$, then

$$
e_{n_{1}}^{(p)} e_{n_{2}}^{(p)} \cdots e_{n_{k}}^{(p)}=0 \text { in } H^{2 n}\left(X, \mathbb{F}_{p}\right) .
$$

As an immediate consequence, we obtain the following result.

Corollary 3. Let $p$ be a prime and $E \rightarrow X$ an oriented $\Sigma_{g}$-bundle over a closed oriented $2 n$-manifold $X$. If $n$ is odd and satisfies $n \equiv-1$ $(\bmod p-1)$, then

$$
e_{n}^{(p)}=0 \text { in } H^{2 n}\left(X, \mathbb{F}_{p}\right)
$$

To prove Theorem 5, we recall a certain relation among MoritaMumford classes and Chern classes which was proved by Morita [28] along the line of Atiyah [2]. Let $\pi: E \rightarrow X$ be a $\Sigma_{g}$-bundle over a closed oriented manifold $X$. For each fiber $E_{x}=\pi^{-1}(x)(x \in X)$, consider the real cohomology $H^{1}\left(E_{x}, \mathbb{R}\right)$. The natural projection

$$
\eta: \bigcup_{x \in X} H^{1}\left(E_{x}, \mathbb{R}\right) \longrightarrow X
$$

gives rise to a $2 g$-dimensional real vector bundle over $X$. A choice of a fiber metric on $\pi$ yields a structure of $g$-dimensional complex vector bundle on $\eta$. Let $c_{n}(\eta) \in H^{2 n}(X, \mathbb{Z})$ be the $n$-th Chern class of $\eta$. Note that $c_{n}(\eta)$ coincides with the pull-back of the $n$-th Chern class $c_{n} \in H^{2 n}\left(\Gamma_{g}, \mathbb{Z}\right)$ of $\Gamma_{g}$ by the holonomy homomorphism $h: \pi_{1}(X) \rightarrow \Gamma_{g}$. Now the AtiyahSinger index theorem for families of elliptic operators or the Grothendieck Riemann-Roch theorem implies

$$
e_{2 n-1}=(-1)^{n} \frac{2 n}{B_{2 n}} s_{2 n-1}(\eta) \text { in } H^{4 n-2}(X, \mathbb{Q})
$$


for all $n$, where $B_{2 n}$ is the $2 n$-th Bernoulli number and $s_{n}(\eta) \in H^{2 n}(X, \mathbb{Z})$ is the characteristic class of $\eta$ corresponding to the formal sum $\sum_{k} x_{k}^{n}$ (it is an integral polynomial of Chern classes of $\eta$ and is called the $n$-th Newton class of $\eta$ ). See [28] for further detail. Now we will prove Theorem 5.

Proof of Theorem 5. Without loss of generality, we may assume $n_{1}$ is odd and satisfies $n_{1} \equiv-1(\bmod p-1)$. For simplicity, set $2 m-1=n_{1}$. It follows from (7.2) that

$$
e_{2 m-1} \prod_{i=2}^{k} e_{n_{i}}=(-1)^{m} \frac{2 m}{B_{2 m}} s_{2 m-1}(\eta) \prod_{i=2}^{k} e_{n_{i}} \text { in } H^{2 n}(X, \mathbb{Q}) .
$$

Let $\operatorname{num}\left(B_{2 m} / 2 m\right)$ (resp. $\left.\operatorname{den}\left(B_{2 m} / 2 m\right)\right)$ be the numerator (resp. denominator) of $B_{2 m} / 2 m$. Then the previous equality leads to

$$
\operatorname{num}\left(\frac{B_{2 m}}{2 m}\right) e_{2 m-1} \prod_{i=2}^{k} e_{n_{i}}=(-1)^{m} \operatorname{den}\left(\frac{B_{2 m}}{2 m}\right) s_{2 m-1}(\eta) \prod_{i=2}^{k} e_{n_{i}}
$$

in $H^{2 n}(X, \mathbb{Q})$. Observe that both sides of (7.3) are integral. In other words, they belong to the image of the homomorphism $H^{*}(X, \mathbb{Z}) \rightarrow H^{*}(X, \mathbb{Q})$ induced by $\mathbb{Z} \hookrightarrow \mathbb{Q}$. Since $H^{2 n}(X, \mathbb{Z})$ is torsion-free, we conclude that $(7.3)$ holds in $H^{2 n}(X, \mathbb{Z})$. As $2 m \equiv 0(\bmod p-1)$, von Staudt's theorem implies that $\operatorname{den}\left(B_{2 m} / 2 m\right)$ is divisible by $p$ (see [3] for instance). Applying the mod $p$ reduction to $(7.3)$, we have

$$
\operatorname{num}\left(\frac{B_{2 m}}{2 m}\right) e_{n_{1}}^{(p)} e_{n_{2}}^{(p)} \cdots e_{n_{k}}^{(p)}=0 \text { in } H^{2 n}\left(X, \mathbb{F}_{p}\right) .
$$

Since $\operatorname{num}\left(B_{2 m} / 2 m\right)$ is prime to $p$, the theorem is proved.

Now let $s_{n} \in H^{2 n}\left(\Gamma_{g}, \mathbb{Z}\right)$ be the $n$-th Newton class of $\Gamma_{g}$ which is defined to be the characteristic class corresponding to the formal sum $\sum_{k} x_{k}^{n}$ of the $g$-dimensional complex vector bundle associated with the continuous map $B \Gamma_{g} \rightarrow B U(g)$ introduced in $\S 5$. Then the Grothendieck Riemann-Roch theorem implies

$$
e_{2 n-1}=(-1)^{n} \frac{2 n}{B_{2 n}} s_{2 n-1} \text { in } H^{4 n-2}\left(\Gamma_{g}, \mathbb{Q}\right)
$$

for all $n \geq 1$. In view of the proof of Theorem 5 , it is valuable to propose the following conjecture. 
Conjecture 3 .

$$
\operatorname{num}\left(\frac{B_{2 n}}{2 n}\right) e_{2 n-1}=(-1)^{n} \operatorname{den}\left(\frac{B_{2 n}}{2 n}\right) s_{2 n-1} \quad \text { in } \quad H^{4 n-2}\left(\Gamma_{g}, \mathbb{Z}\right)
$$

holds for all $n \geq 1$.

According to Proposition 6, Conjecture 3 is affirmative for $n=1$. By virtue of the proof of Theorem 5 , the affirmative solution of Conjecture 3 implies those of Conjectures 1 and 2 for $\bmod p$ Morita-Mumford classes of odd indices. We will deal with Conjecture 3 in the forthcoming paper with N. Kawazumi.

\section{§8. Applications to structure of oriented $\Sigma_{g}$-bundles}

The purpose of this section is to investigate structure of oriented $\Sigma_{g^{-}}$ bundles for $g=2$ or 3 by applying Theorem 9 . Let $\pi: E \rightarrow X$ be an oriented $\Sigma_{g}$-bundle over a closed manifold $X$ which is not necessarily orientable. Then the tangent bundle $T_{*}(E)$ of $E$ decomposes itself into

$$
T_{*}(E)=T_{E / X} \oplus \pi^{*}\left(T_{*}(X)\right) .
$$

Hence the total Stiefel-Whitney class $w .(E)$ of $E$ is expressed as

$$
w \cdot(E)=\left(1+e\left(T_{E / X}\right)\right) \cdot \pi^{*}(w \cdot(X)) \text { in } H^{*}\left(E, \mathbb{F}_{2}\right)
$$

As (7.1) remains valid over $\mathbb{F}_{2}$ and $\left\langle u,[E]_{2}\right\rangle=\left\langle\pi_{!}(u),[X]_{2}\right\rangle$ holds for all $u \in H^{\operatorname{dim} E}\left(E, \mathbb{F}_{2}\right)$, we see that all the Stiefel-Whitney numbers of $E$ are expressed by the Stiefel-Whitney classes of $X$ and the mod 2 Morita-Mumford classes of $\pi$. In particular, if $g=2$ or 3 then it follows from Theorem 9 that all the Stiefel-Whitney numbers of $E$ vanish. Hence we obtain the following result.

Proposition 12. Let $E \rightarrow X$ be an oriented $\Sigma_{g}$-bundle over a closed manifold $X$ which is not necessarily orientable. If $g=2$ or 3 , then $E$ is unoriented null-cobordant.

Now let $\pi: E \rightarrow X$ be an oriented $\Sigma_{g}$-bundle over a closed oriented manifold $X$ so that the total space $E$ admits a natural orientation. Then the total rational Pontryagin class $p .(E)$ of $E$ is expressed as

$$
p \cdot(E)=\left(1+e\left(T_{E / X}\right)^{2}\right) \cdot \pi^{*}(p \cdot(X)) \text { in } H^{*}(E, \mathbb{Q}) \text {. }
$$


Since (7.1) remains valid over the rationals and $\langle u,[E]\rangle=\left\langle\pi_{!}(u),[X]\right\rangle$ holds for all $u \in H^{\operatorname{dim} E}(E, \mathbb{Q})$, we see that all the Pontryagin numbers of $E$ are expressed by the rational Pontryagin classes of $X$ and the rational MoritaMumford classes of odd indices of $\pi$. In particular, we obtain the following result.

Corollary 4. Let $E \rightarrow X$ be an oriented $\Sigma_{2}$-bundle over a closed oriented manifold $X$. Then $E$ is oriented null-cobordant.

Proof. Since $\Gamma_{2}$ is $\mathbb{Q}$-acyclic by a result of Igusa [18], we see that all the rational Morita-Mumford classes of $\pi$ vanish and hence all the Pontryagin numbers of $E$ vanish.

Let $E \rightarrow X$ be an oriented $\Sigma_{3}$-bundle over a closed oriented manifold $X$. If $\operatorname{dim} X \not \equiv 2(\bmod 4)$, then $E$ is oriented null-cobordant by Proposition 12 . In case $\operatorname{dim} X \equiv 2(\bmod 4)$, we obtain the following result.

Corollary 5. Let $E \rightarrow X$ be an oriented $\Sigma_{3}$-bundle over a closed oriented manifold $X$ with $\operatorname{dim} X=4 n+2$ for some $n \geq 1$. Suppose that, for any positive integers $n_{1}, n_{2}, \ldots, n_{k}$ satisfying $n=\sum_{i} n_{i}$,

$$
p_{n_{1}}(X) p_{n_{2}}(X) \cdots p_{n_{k}}(X)=0 \text { in } H^{4 n}(X, \mathbb{Q}) \text {, }
$$

where $p_{n_{i}}(X)$ is the $n_{i}$-th rational Pontryagin class of $X$. Then $E$ is oriented null-cobordant.

Proof. It was proved by Faber [8] that $e_{1}^{2}=0$ and $e_{n}=0$ for $n \geq 2$ in $H^{*}\left(\Gamma_{3}, \mathbb{Q}\right)$. Indeed, according to Looijenga [25], one has

$$
H^{*}\left(\Gamma_{3}, \mathbb{Q}\right) \cong \mathbb{Q}\left[e_{1}\right] /\left(e_{1}^{2}\right)+\mathbb{Q} u
$$

where $u$ is an element of degree 6 . Hence all the Pontryagin numbers of $E$ vanish by the assumption.

As an immediate consequence, we obtain the following result.

Corollary 6 . Let $E \rightarrow X$ be an oriented $\Sigma_{3}$-bundle over a closed oriented manifold $X$ with $\operatorname{dim} X \geq 3$. If all the rational Pontryagin classes of $X$ vanish, then $E$ is oriented null-cobordant. 
In case $E \rightarrow X$ is an oriented $\Sigma_{g}$-bundle over a closed oriented surface $X$, the signature $\sigma(E)$ of the total space $E$ was studied by Meyer [26]. He showed that $\sigma(E)=0$ whenever $g=2$. Corollary 4 is a generalization of this fact. He also showed that for every $g \geq 3$ and every $n \in 4 \mathbb{Z}$ there exists an oriented $\Sigma_{g}$-bundle $E \rightarrow X$ over a closed oriented surface $X$ such that $\sigma(E)=n$ (see [2], [7] and [23] for explicit constructions of such $\Sigma_{g}$-bundles). Hence Corollary 6 is not valid when $\operatorname{dim} X=2$.

Remark 6 . Since $e_{1}^{(2)}=0$ for all $g \geq 2$ by Corollary 2, the total space of any oriented $\Sigma_{g}$-bundle over a closed surface which is not necessarily orientable is unoriented null-cobordant.

Acknowledgements. The author thanks to Professor Nariya Kawazumi and Professor Mikio Furuta for valuable comments and discussions with the author.

\section{REFERENCES}

[1] T. Akita, N. Kawazumi and T. Uemura, Periodic surface automorphisms and algebraic independence of Morita-Mumford classes, J. Pure Appl. Algebra, 160 (2001), $1-11$.

[2] M. F. Atiyah, The signature of fibre-bundles, Global Analysis (Papers in Honor of K. Kodaira), Univ. Tokyo Press, Tokyo, 1969, pp. 73-84.

[3] A. I. Borevich and I. R. Shafarevich, Number theory, Academic Press, New York, 1966.

[4] K. S. Brown, Cohomology of Groups, Springer-Verlag, New York, 1982.

[5] F. R. Cohen, Homology of mapping class groups for surfaces of low genus, The Lefschetz centennial conference, Part II (Mexico City, 1984), Contemp. Math. 58, II, Amer. Math. Soc., Providence (1987), pp. 21-30.

[6] C. J. Earle and J. Eells, A fibre bundle description of Teichmüller theory, J. Differential Geometry, 3 (1969), 19-43.

[7] H. Endo, A construction of surface bundles over surfaces with non-zero signature, Osaka J. Math., 35 (1998), 915-930.

[8] C. Faber, Chow rings of moduli spaces of curves. I. The Chow ring of $\overline{\mathcal{M}}_{3}$, Ann. of Math. (2), 132 (1990), 331-419.

[9] C. Faber and E. Looijenga (eds.), Moduli of Curves and Abelian Varieties, The Dutch Intercity Seminar on Moduli, Aspects of Mathematics, E33, Friedr. Vieweg \& Sohn, Braunschweig, 1999.

[10] H. H. Glover, G. Mislin and Y. Xia, On the Yagita invariant of mapping class groups, Topology, 33 (1994), 557-574.

[11] R. Hain and E. Looijenga, Mapping class groups and moduli spaces of curves, Algebraic geometry-Santa Cruz 1995, Proc. Sympos. Pure Math., 62, Part 2, Amer. Math. Soc., Providence (1997), pp. 97-142. 
[12] J. L. Harer, The second homology group of the mapping class group of an orientable surface, Invent. Math., 72 (1983), 221-239.

[13] Stability of the homology of the mapping class groups of orientable surfaces, Ann. of Math. (2), 121 (1985), 215-249.

[14] The virtual cohomological dimension of the mapping class group of an orientable surface, Invent. Math., 84 (1986), 157-176.

[15] - The cohomology of the moduli space of curves, Theory of moduli (Montecatini Terme, 1985), Lecture Notes in Math., 1337, Springer, Berlin (1988), pp. 138-221.

[16] — The third homology group of the moduli space of curves, Duke Math. J., 63 (1991), 25-55.

[17] W. J. Harvey, Cyclic groups of automorphisms of a compact Riemann surface, Quart. J. Math. Oxford Ser. (2), 17 (1966), 86-97.

[18] J.-I. Igusa, Arithmetic variety of moduli for genus two, Ann. of Math. (2), 72 (1960), 612-649.

[19] N. V. Ivanov, On the homology stability for Teichmüller modular groups: closed surfaces and twisted coefficients, Mapping class groups and moduli spaces of Riemann surfaces (Göttingen, 1991/Seattle, WA, 1991), Contemp. Math., 150, Amer. Math. Soc., Providence (1993), pp. 149-194.

[20] N. Kawazumi, Homology of hyperelliptic mapping class groups for surfaces, Topology Appl., 76 (1997), 203-216.

[21] N. Kawazumi and T. Uemura, Riemann-Hurwitz formula for Morita-Mumford classes and surface symmetries, Kodai Math. J., 21 (1998), 372-380.

[22] S. P. Kerckhoff, The Nielsen realization problem, Ann. of Math. (2), 117 (1983), 235-265.

[23] K. Kodaira, A certain type of irregular algebraic surfaces, J. Analyse Math., 19 (1967), 207-215.

[24] R. Lee and S. H. Weintraub, Cohomology of $\mathrm{Sp}_{4}(\mathbf{Z})$ and related groups and spaces, Topology, 24 (1985), 391-410.

[25] E. Looijenga, Cohomology of $\mathcal{M}_{3}$ and $\mathcal{M}_{3}^{1}$, Mapping class groups and moduli spaces of Riemann surfaces (Göttingen, 1991/Seattle, WA, 1991), Contemp. Math., 150, Amer. Math. Soc., Providence (1993), pp. 205-228.

[26] W. Meyer, Die Signatur von Flächenbündeln, Math. Ann., 201 (1973), 239-264.

[27] E. Y. Miller, The homology of the mapping class group, J. Differential Geom., 24 (1986), 1-14.

[28] S. Morita, Characteristic classes of surface bundles, Invent. Math., 90 (1987), 551-577.

[29] Characteristic classes of surface bundles and bounded cohomology, A fête of topology, Academic Press, Boston (1988), pp. 233-257.

[30] Mapping class groups of surfaces and three-dimensional manifolds, Proceedings of the International Congress of Mathematicians, Vol. I, II (Kyoto, 1990), Math. Soc. Japan, Tokyo (1991), pp. 665-674.

[31] - The structure of the mapping class group and characteristic classes of surface bundles, Mapping class groups and moduli spaces of Riemann surfaces (Göttingen, 
1991/Seattle, WA, 1991), Contemp. Math., 150, Amer. Math. Soc., Providence (1993), pp. 303-315.

[32] — Characteristic classes of surface bundles and the Casson invariant, Sugaku Expositions, 7 (1994), 59-79.

[33] Problems on the structure of the mapping class group of surfaces and the topology of the moduli space of curves, Topology, geometry and field theory, World Sci. Publishing, River Edge (1994), pp. 101-110.

[34] - Structure of the mapping class groups of surfaces: a survey and a prospect, Proceedings of the Kirbyfest (Berkeley, CA, 1998), Geometry \& Topology Monographs 2, Geometry \& Topology, Coventry (1999), 349-406.

[35] D. Mumford, Towards an enumerative geometry of the moduli space of curves, Arithmetic and geometry, Vol. II, Progr. Math., 36, Birkhäuser Boston, Boston (1983), pp. 271-328.

[36] D. Quillen, The spectrum of an equivariant cohomology ring. II, Ann. of Math. (2), 94 (1971), 573-602.

[37] P. Symonds, The cohomology representation of an action of $C_{p}$ on a surface, Trans. Amer. Math. Soc., 306 (1988), 389-400.

[38] T. Uemura, Morita-Mumford classes on finite cyclic subgroups of the mapping class group of closed surfaces, Hokkaido Math. J., 28 (1999), 597-611.

[39] Y. Xia, The p-torsion of the Farrell-Tate cohomology of the mapping class group $\Gamma_{(p-1) / 2}$, Topology '90 (Columbus, OH, 1990), Ohio State Univ. Math. Res. Inst. Publ., 1, de Gruyter, Berlin (1992), pp. 391-398.

Department of Mathematics

Hokkaido University

Sapporo, 060-0810

Japan

akita@math.sci.hokudai.ac.jp 